\title{
Hydrodynamical simulations of relativistic astrophysical jets
}

\author{
I.P. van der Westhuizen*, B. van Soelen, P.J. Meintjes \\ Department of Physics, University of the Free State, Bloemfontein, 9301, South Africa \\ E-mail: VanDerWesthuizenIP@ufs.ac.za, VanSoelenB@ufs.ac.za, \\ MeintjPJQufs.ac.za
}

\begin{abstract}
The continuous advance in computing technology enables the implementation of more complex analyses in numerical codes, which allows more realistic simulation of astrophysical processes. In this paper the opensource magnetohydrodynamical code PLUTO is used to create a numerical model for an ideal relativistic jet. The presence of relativistic jets have been found in a variety of astrophysical sources such as gamma-ray bursts and radio-loud active galactic nuclei. The PLUTO code includes numerical Riemann solvers which provide solutions to the fluid dynamical partial differential conservation equations on a structured mesh and allows us to evolve the environment with time. Our initial model consists of a 3-dimensional grid containing a uniform background medium. Relativistic material is injected into the grid from the $z=0$ boundary. The simulation results show the formation of a jet-like structure containing a collimated relativistic central beam surrounded by an outer cocoon of backflow material. Large-scale turbulence was found in the cocoon of the jet which led to the formation of asymmetric structures. The results obtained in the simulation are consistent with those seen in previous studies of ideal relativistic jets and in future models we can now implement more complex effects such as gravity in order to create more realistic models.
\end{abstract}

$3 r d$ Annual Conference on High Energy Astrophysics in Southern Africa-HEASA2015,

18-20 June 2015

University of Johannesburg, Auckland Park, South Africa

\footnotetext{
* Speaker.
} 


\section{Introduction}

The presence of relativistic jets has been found in a variety of sources ranging from Mpc scale jets observed in radio-loud Active Galactic Nuclei (AGN) to short-lived gamma-ray bursts. These jets consist of collimated flows of ejected plasma moving at relativistic speeds and carry large amounts of energy and momentum away from their hosts. Particle acceleration and the subsequent cooling inside relativistic jets, through processes such as synchrotron emission and inverse Compton scattering, make them prominent sources of multi-wavelength emission. High resolution radio mapping of AGN jets revealed complex internal structures such as radio knots and blobs propagating through the jet. The emission observed from these structures must have a direct correlation to the physical environment inside the jet and, therefore, understanding the morphology of relativistic jets is vital. Along with this, the mechanisms for energy transport, the formation, and the collimation of relativistic jets over larger distances are not yet fully understood and is still a topic of ongoing research [1].

Due to the complex nature of the processes that occur within relativistic fluids a purely analytical approach will not be able to produce a complete model for a relativistic jet, instead numerical analysis can provide a powerful tool to produce predictions from current theoretical models that one can compare with observational data. Numerical modelling enables the study of relativistic jet morphology by simulating the predicted environment to determine structural formation inside such a jet. Recent studies in this field focussed on jet instabilities, shock propagation in jets and the influence of magnetic fields on the dynamic structure of relativistic jets [2, 3, 4]. In this paper we present an ideal relativistic hydrodynamic jet simulation, which was created in order to test the capabilities of the magnetohydrodynamical code PLUTO for future projects. In Section 2 of this paper the PLUTO code will be discussed further. Section 3 contains the details of the hydrodynamical model while Section 4 will show the initial results that were obtained. Finally we will end with a short conclusion in Section 5.

\section{The PLUTO code}

PLUTO $^{1}$ is an opensource modular relativistic magnetohydrodynamical numerical code specifically designed for the simulation of supersonic time-dependent flows containing discontinuities. The code integrates a set of general conservation equations of the form

$$
\frac{\partial \vec{U}}{\partial t}=-\nabla \cdot \overrightarrow{\vec{T}}(\vec{U})+\overrightarrow{\vec{S}}(\vec{U})
$$

where $\vec{U}$ is a column vector consisting of conserved quantities, $\overrightarrow{\vec{T}}(\vec{U})$ is a tensor containing the flux vectors as a function of $\vec{U}$, and $\overrightarrow{\vec{S}}(\vec{U})$ is a tensor containing source terms that can be used to introduce effects such as viscosity and gravitational forces [5].

The current iteration of PLUTO supports four different physics modules namely hydrodynamics (HD), relativistic hydrodynamics (RHD), magnetohydrodynamics (MHD) and relativistic magnetohydrodynamics (RMHD) for computation in the different regimes. The different modules incorporate different regimes by changing the components of the conservation vector $\vec{U}$. In

\footnotetext{
${ }^{1}$ The code was implemented by [5] and is available at http://plutocode.ph.unito.it/.
} 
the RHD module, which is used for this simulation, the constituents of equation (2.1) have the following form

$$
\vec{U}=\left[\begin{array}{c}
\rho \Gamma \\
\rho \Gamma^{2} h \mathbf{v} \\
\rho \Gamma^{2} h-P
\end{array}\right], \overrightarrow{\vec{T}}(\vec{U})=\left[\begin{array}{c}
\rho \Gamma \mathbf{v} \\
\rho \Gamma^{2} h \mathbf{v} \mathbf{v}+P \mathbf{I} \\
\rho \Gamma^{2} h \mathbf{v}
\end{array}\right], \overrightarrow{\vec{S}}(\vec{U})=0
$$

where $\rho$ is the proper density, $P$ is the pressure, $h$ is the specific enthalpy, $\Gamma$ is the Lorentz factor, $\mathbf{I}$ is a $3 \times 3$ unit matrix and $\mathbf{v}$ is the three velocity.

The numerical integration of the conservation equations in PLUTO is done using a High Resolution Shock Capturing (HRSC) algorithm with finite volume formalism [5]. This process follows three general steps: firstly the boundary values for each cell are interpolated based on assigned centre average values; A Riemann problem is then set up using the interpolated boundary values and solved using a numerical Riemann solver and finally the solution to the Riemann problem is evolved with time. For our simulations the HLLC Riemann solver was used together with piecewise parabolic interpolation.

\section{Hydrodynamical model}

For the initial model we considered a relativistic jet composed of an ideal fluid, that is a fluid which has zero viscosity and thus no internal friction. The fluid dynamical approach provides an appropriate model for simulating the morphology of relativistic jets even though the collisional mean free path of the particles in the jet is very large. This is because the Debye radius of the constituents are much smaller than the radius of the jet [6]. For simplicity, we considered the case where the jet is dominated by the kinetic energy of the ejected material and the magnetic field plays no dynamical role. This allows us to describe the motion of the jet using purely relativistic hydrodynamic conservation laws as described in Equations (2.1) and (2.2).

The model consisted of a 64x64x64 Cartesian mesh grid in which the unit length represents the radius of the injection nozzle. Arbitrary units were used for all quantities in the simulation since the very large or small values of cgs units would lead to additional numerical errors. A uniform stationary background medium was initially assigned to the mesh grid and a circular injection nozzle was created on the $z=0$ boundary. The injection nozzle was used to inject a steady flow of jet material with a velocity directed along the $z$-axis. In order to simulate the production of bipolar jets a reflective boundary condition was used for the $z=0$ boundary. All other boundary conditions were set to outflow, with a zero gradient across the boundary $\left(\frac{\partial q}{\partial n}=0\right.$, where $q$ represents the conserved variables and $n$ the direction orthogonal to the boundary), such that matter could escape freely.

Four variables were used in order to describe the model; the Lorentz factor $\Gamma$, the Mach number $M_{b}$, the jet to ambient density ratio $\eta$ and the adiabatic index $\gamma_{a d}$. All other jet properties, such as the pressure, the energy and the velocity of the fluid, can be determined from the above mentioned variables [7]. For example the pressure of the system can be solved by combining the ideal caloric equation of state,

$$
e=\frac{P}{\rho\left(\gamma_{a d}-1\right)},
$$




\begin{tabular}{lcc}
\hline Parameter & & $\begin{array}{c}\text { Value } \\
\text { (arbitrary units) }\end{array}$ \\
\hline Lorentz factor & $\Gamma$ & 10 \\
Velocity & $v$ & $0.995 c$ \\
Density ratio & $\eta$ & $10^{-3}$ \\
Jet density & $\rho_{b}$ & 0.1 \\
Ambient density & $\rho_{a m}$ & 100 \\
Mach number & $M_{b}$ & 7.8 \\
Adiabatic index & $\gamma_{a d}$ & $5 / 3$ \\
Pressure & $P$ & 0.001 \\
\hline
\end{tabular}

Table 1: Parameters used in the set up of the initial conditions.

where $e$ is the internal energy of the fluid, with the definition of the sound speed $\left(C_{s}=v / M_{b}\right)$ in a fluid, yielding

$$
P=\frac{\left(\gamma_{a d}-1\right) \rho C_{s}^{2}}{\gamma_{a d}\left(\gamma_{a d}-1-C_{s}^{2}\right)} .
$$

The parameters used for the relativistic jet model are given in Table 1 along with the calculated primary quantities. These parameters were chosen to be similar to previous studies such as $[4,7,8]$. For our model the density of the injected material was normalized to $\rho=1$ in the observer frame and hence a proper density of $\rho=0.1$ was used in the co-moving reference frame. A uniform pressure was used between the background and jet material in accordance with the model presented in [6].

\section{Results}

The simulation described in the previous section was run with two different resolution configurations. For the first case a static resolution of 8 points per unit length was used. Adaptive Mesh Refinement (AMR) was used for the second case, amounting to an equivalent resolution of 16 points per unit length. The AMR dynamically changes the amount of computational cells on the mesh grid depending on the boundary values of adjacent cells in order decrease the computational intensiveness of the simulation. The AMR does this in a way that allows low resolution for regions with minimal activity and high resolution for active regions where more detail is necessary.

Figure 1 shows a 3 -dimensional rendering of the injected material at $t=150$ in arbitrary units for the static grid case. The figure shows a collimated central region, containing only injected material, propagating through the grid. This region is considered as the central beam of the jet model. The central beam of the jet is surrounded by an outer cocoon region containing diluted jet material mixing with the ambient medium.

In order to investigate the morphology of the jet model in detail, 2-dimensional slices through the origin were plotted of the proper density, the pressure and the velocity component in the $z$ direction. The results at different time steps are displayed in Figures 2 and 3 for the static grid and AMR simulations respectively. The figures show that the injection of material at the base of the 


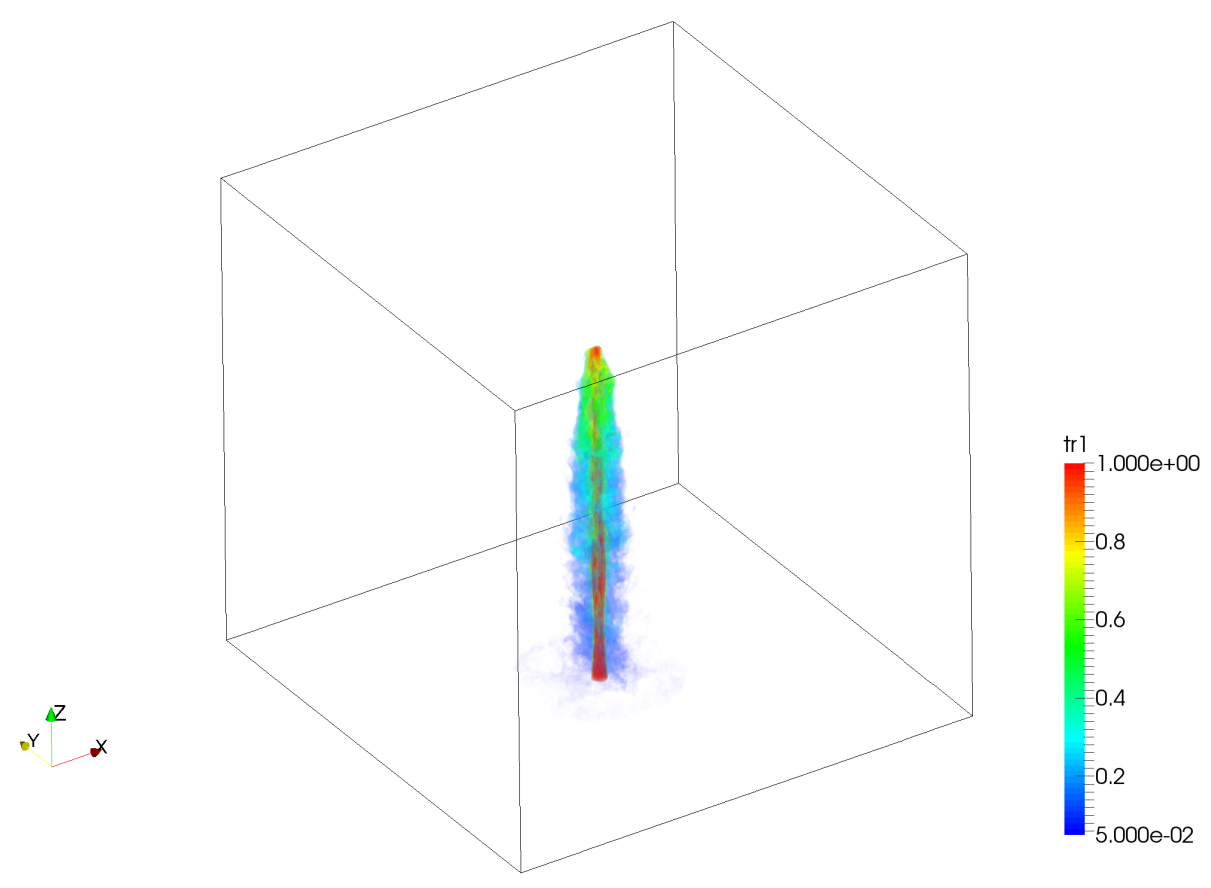

Figure 1: A 3-dimensional rendering showing the trace of the injected material at time $t=150$.

$z$-axis causes a terminal bow shock to form and propagate through the ambient medium. Initially this bow wave appears symmetric due to the uniform nature of the background medium. However, at later stages asymmetry develops as a result of the turbulence inside the cocoon of the jet. As the bow shock propagates through the background medium it compresses the material to form a region of higher pressure between the jet material and the bow wave.

At the front of the jet on the surface between the high density shocked background medium and the low density injected jet material a contact discontinuity, called the working surface, is formed [7]. At the working surface the interaction between the background medium and the jet material converts the kinetic energy of the injected material into internal energy. This interaction also drives a turbulent backflow of material around the central beam of the jet. The backflow material forms the cocoon layer between the shocked background medium and the central beam of the jet.The higher pressure of the shocked ambient medium and the cocoon helps to keep the central beam collimated throughout the simulation [9]. This pressure mismatch between the cocoon of the jet and the injected material also causes periodic shock waves to form inside the central beam of the jet. No large-scale instabilities formed inside the beam of the jet in either of the simulations and the injected material remains well collimated throughout the entire simulation, even at very large timescales such as $t=4300$ when the working surface has left the computational domain. Our simulation showed little deceleration of the material in the central beam since the interaction of the injected material and the background medium is limited by the surrounding cocoon [7]. At larger timescales $(t=4300)$ a thin shear layer forms between the cocoon and the relativistic jet. This layer contains material moving in the positive $z$-direction at sub relativistic speeds $v \approx 0.30 c$. 


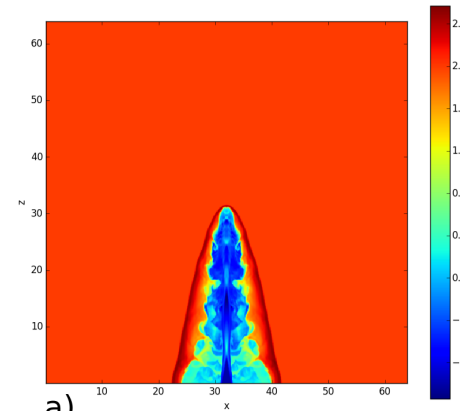

a)
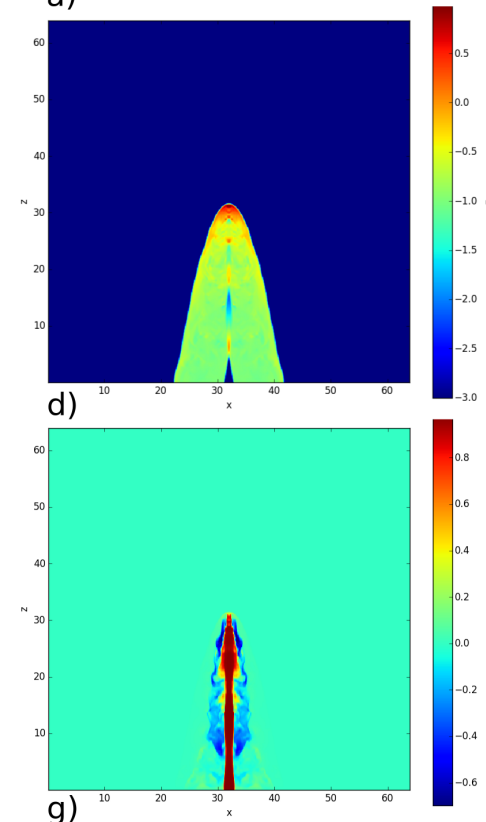

g)

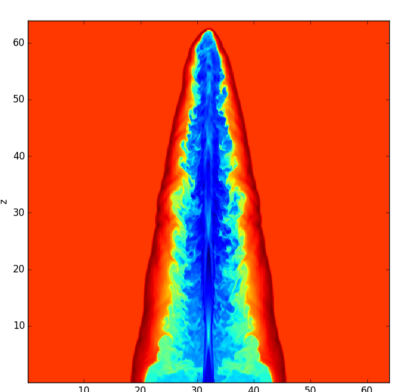

b)

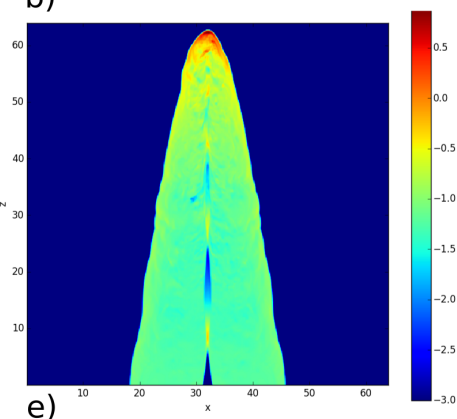

e)

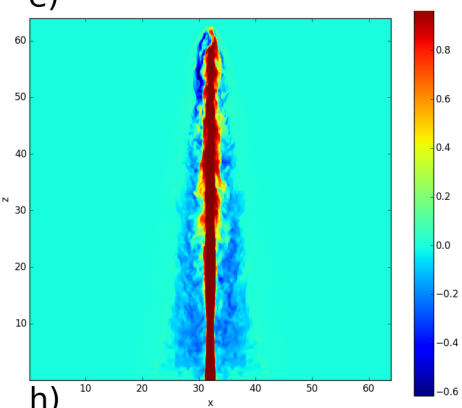

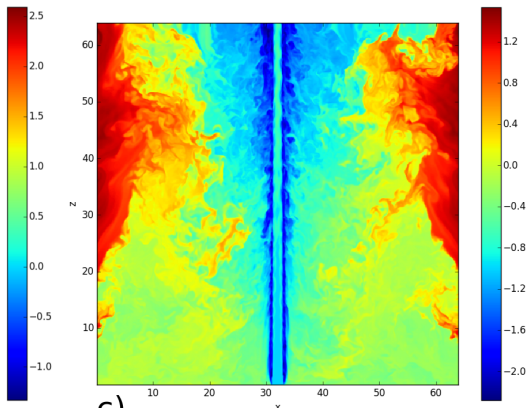

c)
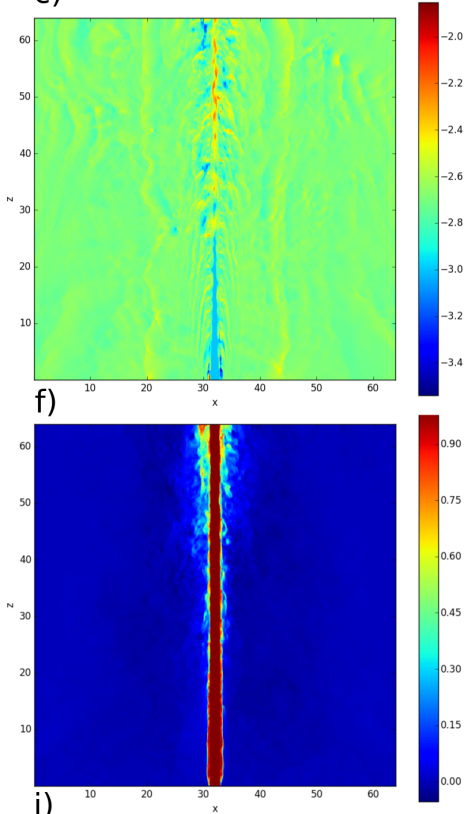

Figure 2: Two-dimensional slices of the static grid simulation, with 8 points per beam width resolution, showing the proper density at (a) $t=100$, (b) $t=200$, (c) $t=4300$; the pressure at (d) $t=100$, (e) $t=200$, (f) $t=4300$, and the proper velocity component in the $z$ direction at (g) $t=100$, (h) $t=200$, (i) $t=4300$. Logarithmic scales are shown for the density and pressure plots in arbitrary units, while the velocity plots have a linear scaling in units of $c$.

Comparing the results in Figure 2 to those of Figure 3 it was found that the AMR simulation yielded a similar morphology to that of the static grid. The AMR simulation however shows a difference in the asymmetry which we attribute to the higher equivalent resolution.

\section{Discussion and conclusion}

A 3-dimensional model for an ideal relativistic jet was set up and evolved with time using the PLUTO magnetohydrodynamical code. The results obtained from this simulation showed the formation of a relativistic collimated central beam with little deceleration surrounded by a cocoon of backflowing material caused by the interaction of injected material and the background medium at the working surface. The formation of these structures were consistent to the morphology shown by previous authors. Some minor differences were found when compared to the previous studies 


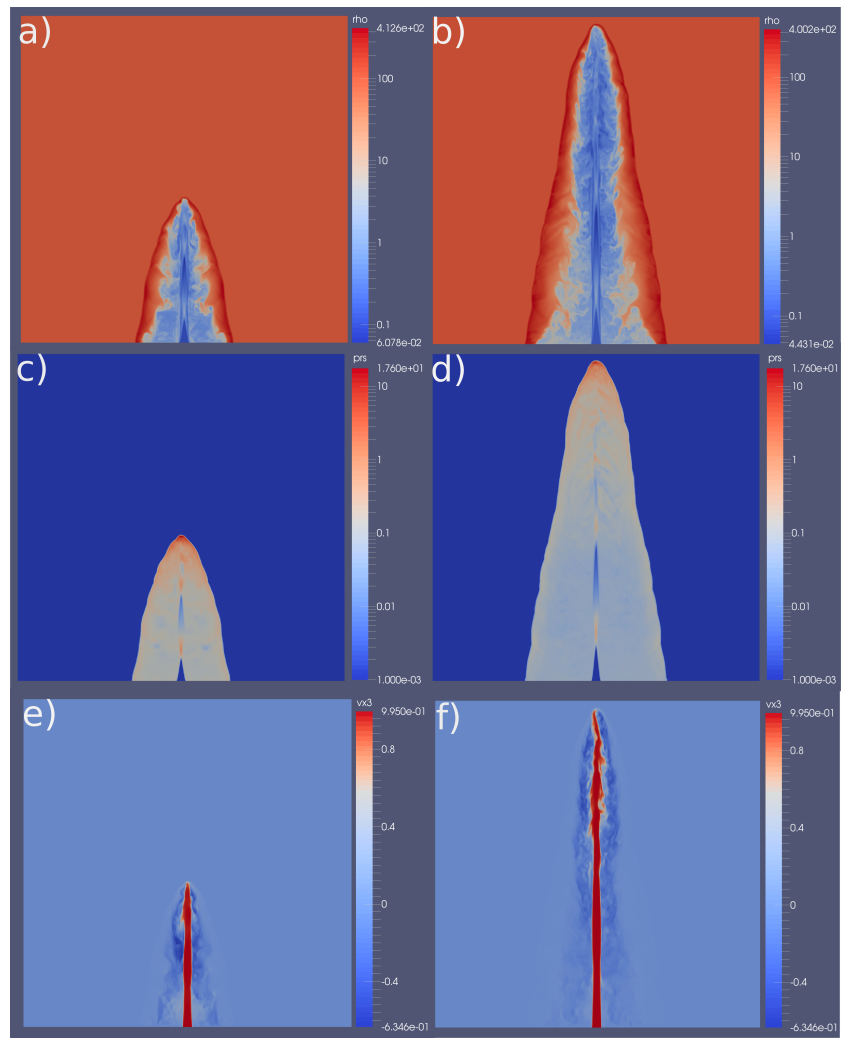

Figure 3: Two-dimensional slices of the AMR simulation, with a 16 points per beam width equivalent resolution, showing the proper density at (a) $t=100$, (b) $t=200$; the pressure at (c) $t=100$, (d) $t=200$, and the proper velocity component in the $z$ direction at (e) $t=100$, (f) $t=200$. Logarithmic scales are shown for the density and pressure plots in arbitrary units, while the velocity plots have a linear scaling in units of $c$.

in the formation of asymmetric turbulence which we attribute to variations in the model setup that was used.

Our future aim is to incorporate more complex effects into this model, that would be present in real relativistic jets, such as gravity, viscosity and magnetic fields. We intend on constructing an emission model which can be used to compared simulated emission to observational data to investigate the effects of variable flow rates, blob production and collision, and a non-uniform background on the emission.

\section{Acknowledgments}

Acknowledgement is given to the High Performance Cluster (HPC) unit at the University of the Free State for their assistance in the installation and set up of software used to run simulations on the UFS HPC.

The financial assistance of the National Research Foundation (NRF) towards this research is hereby acknowledged. Opinions expressed and conclusions arrived at, are those of the author and are not necessarily to be attributed to the NRF. 
This work is based on the research supported in part by the National Research Foundation of South Africa for the grant 87919. Any opinion, finding and conclusion or recommendation expressed in this material is that of the authors and the NRF does not accept any liability in this regard.

\section{References}

[1] Böttcher M, Harris D E and Krawczynski H, Introduction and Historical Perspective; in Relativistic Jets from Active Galactic Nuclei (eds M. Böttcher, D. E. Harris and H. Krawczynski), Wiley-VCH Verlag GmbH \& Co. KGaA, chap 1, pp 1-16 Weinheim, Germany, 2012.

[2] Mignone A, Rossi P, Bodo G, Ferrari A, Massaglia S High-resolution 3D relativistic MHD simulations of jets MNRAS 402, 7-12, 2010.

[3] Mimica P, Aloy M A, Müller E Internal shocks in relativistic outflows: collisions of magnetized shells $A \& A 466$ 93-106, 2007.

[4] Rossi P, Mignone A, Bodo G, Massaglia S and Ferrari A Formation of dynamical structures in relativistic jets: the FRI case A\&A $\mathbf{4 8 8}$ 795-806, 2008.

[5] Mignone A, Bodo G, Massaglia S, Matsakos T, Tesileanu O, Zanni C and Ferrari A PLUTO: a numerical code for computational astrophysics ApJ 170 228-42, 2007.

[6] Blandford R D, Rees M F, A twin-exhaust model for double radio sources, MNRAS 169, 395-415, 1974.

[7] Marti J M, Müller E, Font J A, Ibáñez J M and Marquina Morphology and dynamics of relativistic jets ApJ 479 151-163, 1997.

[8] Aloy M A, Ibáñez J M, Marti J M, Góḿez J L, and Müller E High-resolution three-dimensional simulations of relativistic jets ApJ 523, 125-128, 1999.

[9] Begelman M C, Blandford R D and Rees M J Theory of extragalactic radio sources Rev. Mod. Phys. 56, 255-351, 1984. 